\title{
Cardiovascular Risk Factors in Portuguese Obese Children and Adolescents: Impact of Small Reductions in Body Mass Index Imposed by Lifestyle Modifications
}

\author{
Henrique Nascimento ${ }^{1,2}$, Elísio Costa ${ }^{3}$, Petronila Rocha-Pereira ${ }^{4}$, Carla Rego ${ }^{5}$, Helena Ferreira \\ Mansilha $^{6}$, Alexandre Quintanilha ${ }^{2,7}$, Alice Santos-Silva ${ }^{1,2}$ and Luís Belo*,1,2 \\ ${ }^{I}$ Departamento de Bioquímica, Faculdade de Farmácia, Universidade do Porto, 4050-047 Porto, Portugal \\ ${ }_{2}^{2}$ Instituto de Biologia Molecular e Celular (IBMC), Universidade do Porto, 4150-180 Porto, Portugal \\ ${ }^{3}$ Instituto de Ciências da Saúde, Universidade Católica Portuguesa, Rua Dr. António Bernardino de Almeida, \\ P-4200-072 Porto, Portugal \\ ${ }^{4}$ Centro de Investigação em Ciências da Saúde, Universidade da Beira Interior, 6200-506 Covilhã, Portugal \\ ${ }^{5}$ Unidade de Nutrição / Serviço de Pediatria. UAG-MC. Hospital de S. João E.P.E. Faculdade de Medicina, \\ Universidade do Porto; 4200-319 Porto, Portugal \\ ${ }^{6}$ Hospital Crianças Maria Pia - Centro Hospitalar do Porto; 4050-111 Porto, Portugal \\ ${ }^{7}$ Instituto de Ciências Biomédicas Abel Salazar (ICBAS), Universidade do Porto, 4099-003 Porto, Portugal
}

\begin{abstract}
Objectives: Evaluate cardiovascular risk factors in Portuguese obese children and adolescents and the long-term effects of lifestyle modifications on such risk factors.

Design: Transversal cohort study and longitudinal study.

Setting: University Hospital S. João and Children’s Hospital Maria Pia, Porto.

Patients/Participants: 148 obese children and adolescents [81 females (54.7\%); mean age of 11.0 years] and 33 controls (sex and age matched) participated in a cross-sectional study. Sixty obese patients agreed to participate in an one year longitudinal study after medical and nutritionist appointments to improve lifestyle modification; a substantial body mass index (BMI) reduction was defined by a decrease in BMI z-score (BMI z-sc) of 0.3 or more over the studied period.

Main Outcome measures: Lipid profile (triglycerides, cholesterol, HDLc, LDLc, lipoprotein (a), apolipoproteins A and B) and circulating levels of C-reactive protein (CRP), adiponectin, glucose, and insulin.

Results: Compared with the lean children, obese patients demonstrated statistically significantly higher insulin resistance index [Homeostasis model assessment (HOMA)], and triglycerides, LDLc, apolipoprotein (apo) B, insulin and CRP concentrations, whereas their HDLc and apo A levels were significantly lower (cross-sectional study). In the longitudinal study $(n=60)$, a substantial BMI reduction occurred in $17(28.3 \%)$ obese patients which led to a significant reduction in triglycerides, cholesterol, LDLc, apo B, glucose and insulin levels and in HOMA. The $\triangle \mathrm{BMI}$ values over the studied period correlated inversely and significantly with BMI $(P<0.001)$ and HOMA $(P=0.026)$ values observed at baseline. In multiple linear regression analysis, BMI at baseline remained associated to changes in BMI over the studied period (standardised Beta: $-0.271, P=0.05$ ).

Conclusion: Our data demonstrates that small reductions in BMI-zc, imposed by lifestyle modifications in obese children and adolescents, improve the cardiovascular risk profile of such patients. Furthermore, patients with higher BMI and/or insulin resistance seem to experience a greater relative reduction in their BMI after lifestyle improvements.
\end{abstract}

Keywords: Lipid profile, insulin resistance, inflammation, childhood obesity, lifestyle modifications.

\section{INTRODUCTION}

Obesity is increasing all over the world both in developed and underdeveloped countries $[1,2]$ and is known to be

*Address correspondence to this author at the Departamento de Bioquímica, Faculdade de Farmácia da Universidade do Porto, Rua Aníbal Cunha, 164, 4050-047 Porto, Portugal; Tel: +351-222078906; Fax: +351-222003977; E-mail: luisbelo@ff.up.pt associated with inflammatory changes, insulin resistance and with a hyperlipidemic state [3]. The adipocyte is an important source of cytokines, namely plasminogen activator inhibitor type 1 (PAI)-1, leptin, interleukin (IL)-6 and tumor necrosis factor (TNF)-alpha, and their levels are significantly higher in the plasma of obese patients [4]. Moreover, some inflammatory markers, such as C-reactive protein (CRP), 
show an independent positive association with body mass index (BMI) [5].

Cardiovascular (CV) morbidity and mortality is associated with the classic risk factors, namely dyslipidemia, hypertension and impaired glucose metabolism. However, more recently, inflammatory mechanisms were recognized as playing a vital role in initiation, maintenance and progression of atherosclerotic vascular disease. Atherosclerosis is an inflammatory disease with the participation of leukocytes and several cytokines. Among several inflammatory markers that have been shown to predict CV events [namely IL-6, tumor necrosis factor-alpha and soluble intercellular adhesion molecule (ICAM)-1] CRP levels have emerged as the most powerful marker [6].

Regular physical activity is known to reduce obesityrelated markers and risk of type 2 diabetes (physical activity is associated with improved insulin sensitivity) and to improve endothelial function, lipid profile (e.g., exercise decreases plasma triglycerides (TG) and increases high density lipoprotein (HDL), as well as several oxidative stress/inflammatory markers, all risk factors for $\mathrm{CV}$ disease (CVD) [7-10]. Diet restriction per se may reduce fat mass, total cholesterol (Chol) and low density lipoprotein cholesterol (LDLc) without apparent effect in HDL and limited effect on insulin resistance. The effect of both diet and physical exercise in obese individuals appear to be additive with greater impact in the improvement of lipid profile and insulin resistance [11]. However, few data exists in literature regarding the long-term effects of physical activity on young obese subjects. Moreover, in most studies, children obey to a restricted physical exercise program or a food intake program, but the effect of "natural" lifestyle modifications on $\mathrm{CV}$ risk factors has been poorly explored [12-14].

Higher cardiorespiratory fitness (CRF) has been associated to a decrease in CVD risk markers, CVD morbidity and mortality [15]. Besides physical activity, the initial CRF of an individual appears to be an independent predictor of weight variation. Individuals with lower CRF are more prone to gain weight when compared to those with higher CRF [16]. Actually, children with lower CRF usually present diminished levels of physical activity and increased time expended in sedentary behaviours. Despite the strong genetic influence, CRF status can be modulated through physical activity, which is a main determinant of CRF in pediatric populations [17].

The physical activity performed by children and adolescents in southern European countries is lower than in countries from centre and northern Europe. This fact may explain the geographical differences in obese prevalence found in Europe [17]. In fact, recent studies showed a very high prevalence of overweight/obesity (about 30\%) in Portuguese children when compared to other European countries $[18,19]$. Thus, the study of obesity is of particular importance in our country.

The aim of this study was to evaluate the short-term effects of lifestyle modifications, through a decrease in body mass index z-score (BMI z-sc), on $\mathrm{CV}$ risk factors (circulating levels of C-reactive protein, glucose, insulin and lipid profile), in Portuguese obese children and adolescents.

\section{MATERIAL AND METHODS}

\subsection{Subjects}

The protocol used for all participants was approved by the Committees on Ethics of the University Hospital S. João and of the Children's Hospital Maria Pia, Porto. Obese children and adolescents, aged 5-18 years, were identified from medical records, at the Departments of Pediatrics of the referred hospitals.

Obesity was defined as BMI greater than the $95^{\text {th }}$ percentile for age and gender, according to 2000 Centers for Disease Control and Prevention (CDC) growth charts. Because BMI is not normally distributed and not adjusted for sex and age we used BMI z-sc.

One hundred and forty eight $(n=148)$ obese children and adolescents participated in the study after informed and written consent from their parents. Thirty three healthy control subjects, age and sex matched with obese patients, were recruited from the general population. The BMIs of the controls were lower than the $85^{\text {th }}$ percentile adjusted for sex and age. Obese patients were evaluated at the beginning of the study and compared with controls (cross-sectional study).

All obese individuals $(n=148)$ were motivated to change their lifestyle habits (conventional weight loss programs based on dietary counseling and encouragement to exercise), but only sixty patients agreed to participate in a longitudinal study (one year of follow up). A substantial BMI reduction was defined by a decrease in BMI z-sc of 0.3 or more over the studied period. One year after the beginning of lifestyle modification intervention, obese children/adolescents were classified as: 1) those who have achieved a substantial BMI reduction or 2) those who have not achieved a substantial BMI reduction. In the longitudinal study, clinical and biochemical data were obtained at the beginning as well as at the conclusion, after one year of follow-up.

Criteria of exclusion were: smokers, subjects under regular medication or with diabetes mellitus, endocrinologic disorders, and hereditary, inflammatory or infectious diseases.

\subsection{Procedures and Assays}

\subsubsection{Clinical Examination}

The participants were invited to the research centers after an overnight fast and clinical data regarding the sample population was collected. The development of puberty was clinically assessed on the basis of Tanner stages. The physical examination included the measurement of height, weight and the observation of the presence of cutaneous markers related with obesity and its comorbidity.

\subsubsection{Blood Samples}

Blood samples were obtained on a fasting basis and processed within $2 \mathrm{~h}$ of collection. Blood was obtained by venipuncture in ethylenediaminetetraacetic acid (EDTA) containing tubes. Plasma aliquots were made and immediately stored at $-70^{\circ} \mathrm{C}$ until assayed.

\subsubsection{Laboratory Analysis}

The determination of circulating levels of glucose, insulin, lipids, and lipoproteins was performed by using 
Table 1. Characteristics of the Participants in the Cross-Sectional Study

\begin{tabular}{|c|c|c|c|}
\hline & Controls $(\boldsymbol{n}=\mathbf{3 3})$ & Obese $(\boldsymbol{n}=\mathbf{1 4 8})$ & $\boldsymbol{p}$ \\
\hline \hline Age (years) & $10.2 \pm 3.6$ & $11.0 \pm 3.0$ & 0.173 \\
\hline Sex (female) & $20(60,6 \%)$ & $81(54,7 \%)$ & 0.852 \\
\hline Weight (kg) & $34.7 \pm 12.8$ & $69.0 \pm 22.8$ & $<0.001$ \\
\hline BMI (kg/m $\left.{ }^{2}\right)$ & $17.4 \pm 2.2$ & $29.8 \pm 5.4$ & $<0.001$ \\
\hline BMI z-score & $0.09 \pm 0.68$ & $2.30 \pm 0.44$ & $<0.001$ \\
\hline
\end{tabular}

BMI, body mass index.

Values are given as mean $\pm \mathrm{SD}$, unless otherwise indicated

routine automated technology (Glucose, Insulin, Cholesterol, Triglycerides, HDL Cholesterol Direct, LDL Cholesterol Direct, Apolipoprotein (apo) A1 and apo B, ABX Diagnostics). Lipoprotein (a) (( $\mathrm{Lp}(\mathrm{a}))$ plasma concentration was determinated by an immunoturbidimetric method.

Homeostasis model assessment (HOMA), used to detect the degree of insulin resistance [20], was calculated by using the following formula: resistance $(\mathrm{HOMA})=($ fasting insulin $(\mu \mathrm{U} / \mathrm{mL}) \times$ fasting glucose $(\mathrm{mmol} / \mathrm{L}) / 22.5)$.

Plasma levels of CRP were evaluated by immunoturbidimetry [CRP (latex) High-Sensitivity, Roche Diagnostics].

Plasma concentration of adiponectin was evaluated by using a standard commercial enzyme-linked immunoassay (Adiponectin, R\&D Systems).

\subsection{Statistical Analysis}

Statistical analysis was performed using the Statistical Package for Social Sciences (SPSS, version 17.0) for Windows. Kolmogorov-Smirnov analysis was used to test if the results were normally distributed. Results with normal distribution were presented as mean \pm standard deviation, whilst irregular distributions were presented as median (interquartile range).

To compare obese and control groups, Student's unpaired $t$-test was used for parameters presenting a Gaussian distribution and the Mann-Whitney $U$-test in the case of a non-Gaussian distribution. To evaluate the differences occurring along the longitudinal study, we used the paired Student $t$ test for parametric and Wilcoxon Signed Ranks Test for non-parametric variables.

Spearman's rank correlation coefficient was used to evaluate relationships between sets of data.

To evaluate the contribution of the different variables to BMI reduction over the studied period, we performed multiple regression analysis, using stepwise selection with an entry criteria of $P<0.05$, after $\log$ transformation of the variables (when necessary).

Significance was accepted at $P$ less than 0.05 .

\section{RESULTS}

The characteristics of the participants in the crosssectional study are presented in Table $\mathbf{1}$. Obese patients and controls were matched for age and gender. Compared with the lean children, obese patients demonstrated significantly higher insulin resistance index (HOMA), and TG, LDLc, apo $\mathrm{B}$, insulin and CRP concentrations, whereas their HDLc and apo A levels were significantly lower (Table 2). The participants in the cross-sectional study had BMI which correlated positively and significantly to TG $(r=0.300, P<$ $0.001)$, LDLc $(r=0.235, P=0.001)$, insulin $(r=0.652, P<$ $0.001)$, HOMA $(r=0.646, P<0.001)$, and CRP $(r=0.451$, $P<0.001)$ values, and negatively with HDLc $(r=-0.462, \mathrm{P}$ $<0.001)$. Adiponectin levels $(n=153)$ correlated inversely and significantly with age $(r=-0.299, P<0.001)$, BMI $(r=-$ $0.220, \mathrm{P}=0.006)$, TG $(r=-0.390, P<0.001)$ and total cholesterol/HDLc (Chol/HDLc) ratio $(r=-0.270, P=0.001)$, insulin $(r=-0.211, P=0.009)$ and HOMA $(r=-0.223, P=$ $0.006)$, and correlated positively and significantly with apo A $(r=0.214, P=0.008)$ and HDLc $(r=0.270, P=0.001)$.

Sixty obese patients participated in the longitudinal study and, after one year of follow up, 17 (28.3\%) achieved a substantial BMI z-sc reduction (mean $\triangle \mathrm{BMI}$ z-sc $=-0.46 \pm$ $0.16)$, while 43 individuals did not reach the cut-off value (mean $\triangle \mathrm{BMI}$ z-sc $=0.01 \pm 0.26$ ) (Table 3). At baseline (T1), no significant differences were observed in age, sex, BMI, BMI z-sc, and in any markers of the metabolic syndrome or inflammatory markers between obese children who presented with and without substantial BMI z-sc reduction, at the end of the study (T2).

A substantial decrease in BMI z-sc in 17 children led to a significant reduction in $\mathrm{TG}$, cholesterol, LDLc, apo $\mathrm{B}$, glucose, insulin levels and in HOMA (Table 4). In the 43 children without change of BMI z-sc, a reduction in adiponectin and in glucose values occurred as well as a significantly increase in Lp(a) values (Table 4).

The $\triangle \mathrm{BMI}$ values over the studied period (longitudinal study, $n=60$ ) correlated inversely and significantly with BMI at baseline $(r=-0.487, P<0.001$, Fig. 1A), and HOMA at baseline $(r=-0.291, P=0.026$, Fig. 1B). In multiple linear regression analysis, BMI values at baseline remained associated to changes in BMI over the studied period (standardised Beta: $-0.271, P=0.05$ ).

\section{DISCUSSION}

The present study was able to demonstrate in young obese patients that small reductions in BMI z-sc are associated with significant improvements in lipid profile and in insulin resistance index. 
Table 2. Biochemical Data of the Participants in the Cross-Sectional Study

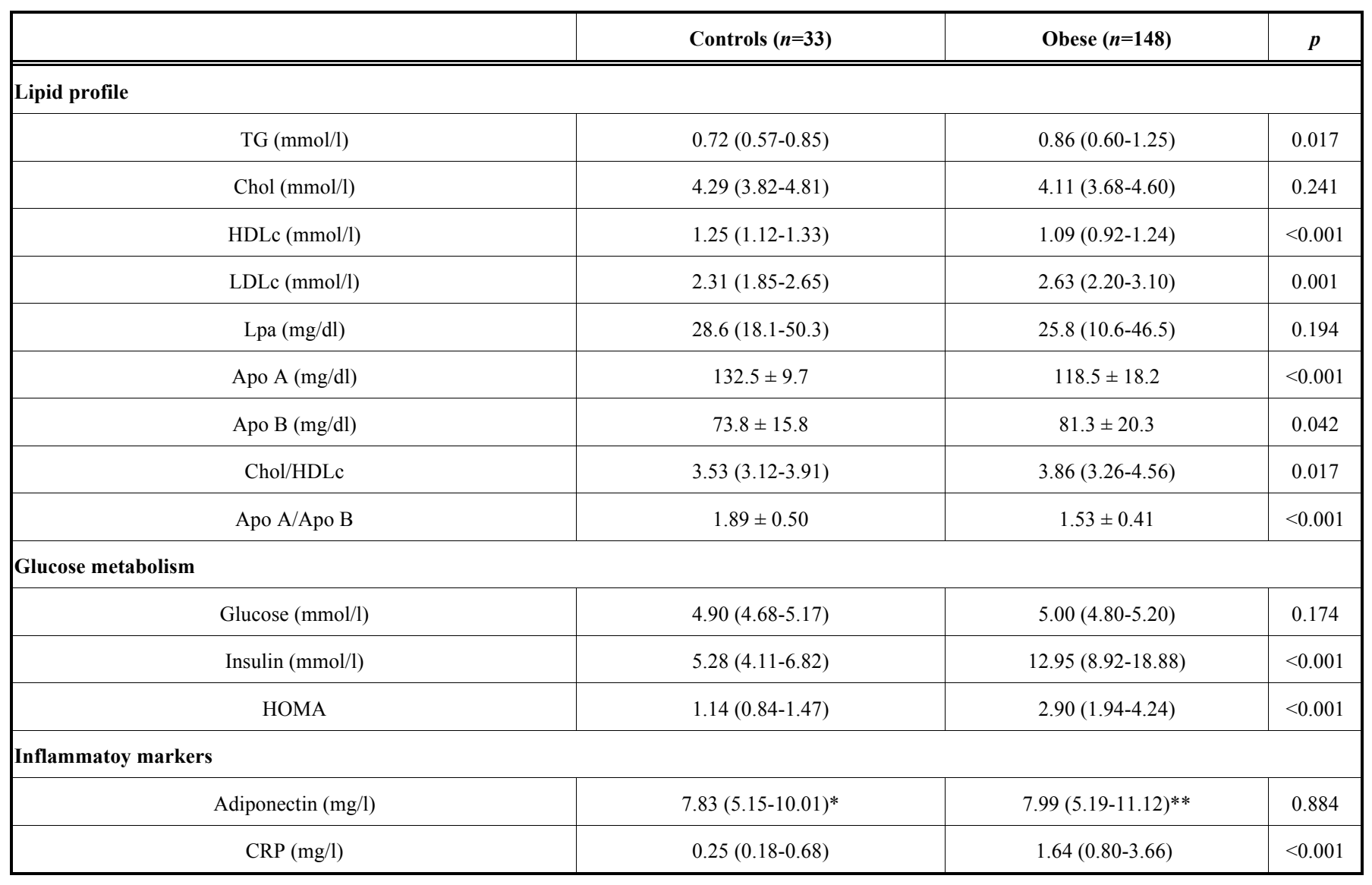

$*, n=14 ; * *, n=139$; Values are given as mean $\pm \mathrm{SD}$ or median (interquartile range).

TG, triglycerides; Chol, cholesterol; HDLc, High Density Lipoprotein cholesterol; LDLc, Low Density Lipoprotein cholesterol; Lp(a), lipoprotein (a); apo, apolipoprotein; HOMA, Homeostasis Model Assessment (insulin resistance index); CRP, C-reactive protein.

Table 3. Characteristics of the Participants in the Longitudinal Study (One Year of Follow-Up)

\begin{tabular}{|c|c|c|c|c|c|c|}
\hline & \multicolumn{2}{|c|}{$\Delta$ BMI z-sc $<0,3(n=43)$} & \multirow[b]{2}{*}{$p$} & \multicolumn{2}{|c|}{$\Delta$ BMI z-sc $\geq 0,3(n=17)$} & \multirow[b]{2}{*}{$p$} \\
\hline & T1 & $\mathbf{T} 2$ & & T1 & $\mathbf{T 2}$ & \\
\hline Weight (kg) & $70.2 \pm 19.1$ & $78.0 \pm 18.0$ & $<0.001$ & $69.9 \pm 16.7$ & $67.5 \pm 13.4$ & 0.191 \\
\hline Waist/Hip & $0.928 \pm 0.054$ & $0.920 \pm 0.065$ & 0.347 & $0.912 \pm 0.028$ & $0.905 \pm 0.059$ & 0.630 \\
\hline BMI $\left(\mathrm{kg} / \mathrm{m}^{2}\right)$ & $29.2 \pm 4.8$ & $30.6 \pm 4.8$ & 0.005 & $30.2 \pm 4.0$ & $27.1 \pm 3.1$ & $<0.001$ \\
\hline BMI z-score & $2.14 \pm 0.36$ & $2.15 \pm 0.32$ & 0.845 & $2.36 \pm 0.68$ & $1.90 \pm 0.65$ & $<0.001$ \\
\hline
\end{tabular}

BMI, body mass index Values are given as mean \pm SD.

In a first analysis (cross-sectional study) we compared obese patients with a lean control group. As some of the studied CV risk factors change with age and are influenced by gender, we matched controls and patients for gender and age. The results obtained confirm obesity as an inflammatory disease. Indeed, the median CRP value observed in obese children and adolescents was about 6 times higher than that observed in controls, demonstrating an inflammatory process in such young patients (Table 2). We also observed that obese patients presented with an altered lipid profile and raised markers of insulin resistance when compared with controls (Table 2).

There are few studies assessing the long-term changes of lifestyle modifications in obese children and adolescents, particularly on $\mathrm{CV}$ risk factors. In the present longitudinal study, obese patients (children and adolescents) were asked to improve their lifestyle habits (conventional weight loss programs based on dietary counselling and encouragement to 
Table 4. Biochemical Data of the Participants in the Longitudinal Study (One Year of Follow-Up)

\begin{tabular}{|c|c|c|c|c|c|c|}
\hline & \multicolumn{3}{|c|}{$\Delta$ BMI z-sc $<0,3(n=43)$} & \multicolumn{3}{|c|}{$\Delta$ BMI z-sc $\geq 0,3(n=17)$} \\
\hline & T1 & $\mathbf{T} 2$ & $p$ & T1 & T2 & $p$ \\
\hline $\mathrm{TG}(\mathrm{mmol} / \mathrm{l})$ & $0.77(0.50-1.02)$ & $0.65(0.51-0.95)$ & 0.795 & $0.87(0.64-1.30)$ & $0.63(0.50-0.90)$ & 0.003 \\
\hline Chol (mmol/l) & $3.93(3.50-4.68)$ & $4.10(3.51-4.50)$ & 0.929 & $4.24(3.80-4.70)$ & $4.00(3.58-4.24)$ & 0.009 \\
\hline LDLc (mmol/l) & $2.59(2.20-3.00)$ & $2.47(2.21-2.90)$ & 0.534 & $2.69(2.30-3.24)$ & $2.42(2.06-2.69)$ & 0.007 \\
\hline Lpa (mg/dl) & $19.0(10.0-43.0)$ & $28.0(12.6-48.6)$ & 0.011 & $22.0(14.0-49.1)$ & $30.60(8.29-51.55)$ & 0.403 \\
\hline Apo A (mg/dl) & $118.5 \pm 18.5$ & $114.1 \pm 18.0$ & 0.052 & $118.3 \pm 22.4$ & $109.8 \pm 17.4$ & 0.060 \\
\hline \multicolumn{7}{|l|}{ Glucose metabolism } \\
\hline Glucose (mmol/l) & $5.00(4.83-5.22)$ & $4.80(4.50-5.10)$ & 0.014 & $5.00(4.64-5.28)$ & $4.57(4.04-4.98)$ & 0.002 \\
\hline Insulin (mmol/l) & $12.70(9.50-15.70)$ & $11.40(9.54-16.60)$ & 0.395 & $14.40(11.60-17.80)$ & $10.90(7.6-14.38)$ & 0.015 \\
\hline HOMA & $2.80(2.14-3.56)$ & $2.33(1.82-3.31)$ & 0.255 & $3.01(2.41-4.24)$ & $1.98(1.51-2.89)$ & 0.007 \\
\hline \multicolumn{7}{|l|}{ Inflammatoy markers } \\
\hline Adiponectin (mg/l) & $8.58(5.56-11.66)$ & $6.04(4.74-9.13)$ & 0.004 & $9.28(7.37-15.02)$ & $10.17(6.63-14.22)$ & 0.675 \\
\hline CRP (mg/l) & $1.61(0.82-4.62)$ & $1.67(0.78-4.12)$ & 0.402 & $0.94(0.43-2.02)$ & $1.07(0.54-2.38)$ & 0.213 \\
\hline
\end{tabular}
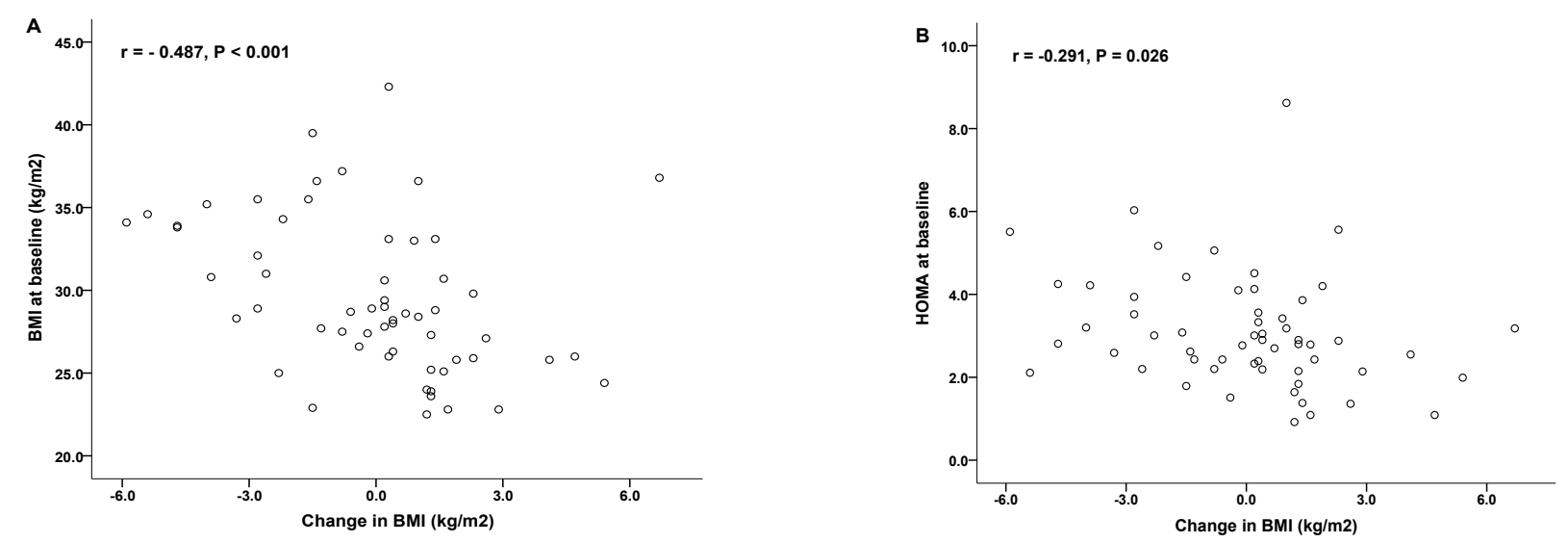

Fig. (1). Associations between change in body mass index (BMI) over the studied period (one year of follow-up) and BMI and insulin resistance index (Homeostasis model assessment; HOMA) values presented by participants at baseline. Spearman's rank correlation coefficient values are shown.

exercise) and an objective measure (BMI z-sc reduction) was used to observe which children achieved the objective after one year of follow-up. We observed that 17 patients $(28.3 \%)$ presented a significant reduction in BMI z-sc $(0.3$ or more $(\triangle \mathrm{BMI} \leq-0.3))$ over the studied period and that changes in BMI over the studied period were independently associated with BMI values presented at baseline. It seems that patients with higher BMI experience a greater relative reduction in their BMI (Fig. 1). Also, the correlation between HOMA in the beginning of the study and relative BMI reduction points to a predisposition for children who are more insulin resistant to lose more weight. These associations can be related to a change in body physiology towards weight loss.

In our longitudinal study, children did not obey to a restricted physical exercise program or a food intake programme. Also, it was not controlled the initial CRF of our population. Thus, it is impossible to know the possible impact of CRF in BMI z-sc reduction. It is true that the lack of standardisation on plans on intervention may mitigate the 
interpretation of the results that we observed. On the other hand, the counseling to improve lifestyle habits may be a "more natural" approach, and is useful in clinical practice, as participants can directly observe the real benefits of changing their lifestyle habits. Furthermore, the reduction in BMI-zc that we observed is likely to express a real change in lifestyle habits.

It was shown that 3 months of moderate lifestyle intervention (45 min of physical activity 3 times per week) in obese adolescents seems to attenuate the inflammatory state associated with obesity, as observed by a reduction in elevated circulating concentrations of CRP, fibrinogen and IL-6, non-traditional risk factors for CVD [12, 14]. Reinehr et al., by evaluating 16 [14] and 10 [13] obese children who lost weight over a 1-year period, found a significant decrease in CRP but no significant changes in TNF-alpha levels. In the studies performed by Reinehr et al., obese children participated in a 1-year obesity intervention program and the cut-off value used for BMI z-sc reduction was 0.5. As we used a smaller cut-off value (0.3), this may explain why we did not observe significant changes in CRP levels. Indeed, CRP shows a positive association with BMI, and our cut-off value may be insufficient to observe clear improvements in CRP. Even thus, it is important to emphasize that in our study, involving a larger number of participants, $64.3 \%$ of the patients that reduced their BMI z-sc presented a decrease in circulating CRP levels, in contrast with the group without change of BMI z-sc (47.6\%). Furthermore, we were able to demonstrate that smaller reductions in BMI $\mathrm{Z}$-sc are associated with significant improvements in lipid profile and in insulin resistance index HOMA. Previous studies suggested that the improvement of insulin sensitivity and CV risk factors in obese children only occurs if BMI $\mathrm{z}$-sc decreases by at least 0.5 over a 1 -year period [21], but our data suggests that such improvements may be observable with smaller reductions in BMI-zc. Again, our results are probably explained by the fact that the reductions that we observed in BMI z-sc are likely to express a continuous daily motivation in changing lifestyle habits. Nevertheless, although there was no change in HDLc, the lower values of HOMA together with the reduction in TG seen in the group that reached the cut-off values leads to the probable influence of concomitant changes in diet and in the levels of physical exercise in this group to be linked with the improvement seen in the global CV risk profile.

The improvement in insulin sensitivity with BMI z-sc reduction observed in our study is of particular importance, as insulin resistance represents a major underlying abnormality driving CVD [22]. It is important to emphasize that insulin resistance associates with increased TG and that one of the most striking findings in the longitudinal analysis of the present study was the reduction in $\mathrm{TG}$ levels. Although hypercholesterolemia is a major risk factor for the initiation and progression of atherosclerosis [23], hypertriglyceridemia and elevated levels of triglyceride (TG)-rich lipoproteins have recently re-emerged as risk factors for atherosclerosis [24]. TG-rich particles may directly damage the endothelium principally via oxidative mechanisms. Also, an important consequence of hypertriglyceridemia, relating to increased atherosclerotic risk, is a shift in the spectrum of LDL subfractions towards smaller, denser species, which are believed to be more atherogenic [25]. Moreover, raised TG levels are frequently associated with reduced HDL levels. In our study, we only observed a slight (non-significant) increase in HDLc levels, which seems to have a protective role in the development of atherosclerosis [26-28]. Reinehr et al. observed a trend to increase HDLc levels in patients who achieved a substantial BMI z-sc reduction [14].

Plasma Lp(a) levels are known to be mainly genetically determined [29, 30]. However, it appears that plasma Lp(a) concentration increases with age. In our study, Lp(a) levels were similar betweens controls and obese individuals but, in the longitudinal study an increase in this lipoprotein was observed (Table 4). In fact, this increase achieved statistically significance in the group without a substantial reduction in BMI z-sc. Thus, our data is in agreement with the idea that ageing elevates plasma Lp(a) concentration, and that this increment might be modulated be lifestyle modifications.

Adiponectin is an adipocytokine that has been noted as an important antiantherogenic, antidiabetic and as an antiinflammatory protein [31]. Adiponectin seems to be inversely related to systolic blood pressure, waist circumference, triglycerides, and 2-hour glucose levels, while powsitively related to HDL [32]. These associations are in agreement with the results obtained in our crosssectional study. However, and although it was suggested that adolescent obesity is associated with low plasma adiponectin levels [33], we were unable to find statistically significant differences in plasma levels of adiponectin between obese children and controls. This may be explained by the fact that we defined controls as having a BMI lower than the $85^{\text {th }}$ percentile adjusted for sex and age, in contrast with other work where control group was defined as a group of adolescents who had a BMI between the $50^{\text {th }}$ and $75^{\text {th }}$ percentiles [33].

Regarding our longitudinal study, it is important to highlight that adiponectin levels decreased significantly in the group without BMI z-sc reduction, in contrast with a moderate (non-significant) increase in the group that presented a significant decrease in BMI z-sc. It was previously reported that a significant weight loss in children, defined as BMI z-sc reduction of 0.5 or more, is associated with a significant increase in adiponectin [34]. Considering the protective effects of adiponectin, it seems reasonable to hypothesize that the increase in adiponectin is likely to explain, at least in part, some of modifications in the lipid profile (namely the reduction in triglycerides) and in glucose metabolism associated with BMI z-sc reduction.

The concern with childhood obesity must begin in overweight children and not only in severe obese children as it is easier to intervene in an early phase. Besides, there are studies that point that the majority of the diseases appear in individuals with moderated elevated risk factors [35].

Further studies involving a standardized physical activity and diet programs together with the assessment of important variables as CRF, at baseline and through the study period, could help to better understand the process involved in weight loss differences found among children and, also, the relation between the changes in adiposity and variations in CVD risk markers. 
In summary, our data demonstrates that lifestyle modifications in obese children and adolescents, even associated with small reductions in BMI z-sc, improve the $\mathrm{CV}$ risk profile, namely lipid profile and insulin resistance. This may be important in motivating patients as health changes can appear earlier than the esthetical ones. Furthermore, patients with higher BMI and/or insulin resistance seem to experience a greater relative reduction in their BMI after lifestyle improvements, probably due to a physiologic adaptation favoring weight loss.

\section{CONFLICT OF INTEREST}

None declared.

\section{ACKNOWLEDGEMENTS}

The authors wish to thank the technicians Amélia Ferreira, Andreia Sousa, Joana Barros and Isabel Almeida for expert assistance on blood collection, and University of Porto and "Fundação para a Ciência e a Tecnologia" (FCT) (SFRH/BD/61407/2009) for financial support.

\section{REFERENCES}

[1] Duncan, S.; Duncan, E.K.; Fernandes, R.A.; Buonani, C.; Bastos, K.D.; Segatto, A.F.; Codogno, J.S.; Gomes, I.C.; Freitas, I.F., Jr., Modifiable risk factors for overweight and obesity in children and adolescents from Sao Paulo, Brazil. BMC Public Health, 2011, 11, 585.

[2] Ko, G.T.; Chan, J.C. Burden of obesity--lessons learnt from Hong Kong Chinese. Obes. Rev., 2008, 9(Suppl 1), 35-40.

[3] Alexander, C.M.; Landsman, P.B.; Grundy, S.M. The influence of age and body mass index on the metabolic syndrome and its components. Diabetes Obes. Metab., 2008, 10(3), 246-250.

[4] Rondinone, C.M. Adipocyte-derived hormones, cytokines, and mediators. Endocrine, 2006, 29(1), 81-90.

[5] Folsom, A.R.; Pankow, J.S.; Tracy, R.P.; Arnett, D.K.; Peacock, J.M.; Hong, Y.; Djousse, L.; Eckfeldt, J. H. Association of Creactive protein with markers of prevalent atherosclerotic disease. Am. J. Cardiol., 2001, 88(2), 112-117.

[6] Blake, G.J.; Ridker, P.M. Novel clinical markers of vascular wall inflammation. Circ. Res., 2001, 89(9), 763-771.

[7] Ignarro, L.J.; Balestrieri, M.L.; Napoli, C. Nutrition, physical activity, and cardiovascular disease: an update. Cardiovasc. Res., 2007, 73(2), 326-340.

[8] Smith, J.K.; Dykes, R.; Douglas, J.E.; Krishnaswamy, G.; Berk, S. Long-term exercise and atherogenic activity of blood mononuclear cells in persons at risk of developing ischemic heart disease. JAMA, 1999, 281 (18), 1722-1727.

[9] Tsai, A.C.; Sandretto, A.; Chung, Y.C. Dieting is more effective in reducing weight but exercise is more effective in reducing fat during the early phase of a weight-reducing program in healthy humans. J. Nutr. Biochem., 2003, 14(9), 541-549.

[10] Verdaet, D.; Dendale, P.; De Bacquer, D.; Delanghe, J.; Block, P.; De Backer, G. Association between leisure time physical activity and markers of chronic inflammation related to coronary heart disease. Atherosclerosis, 2004, 176(2), 303-310.

[11] Ben Ounis, O.; Elloumi, M.; Ben Chiekh, I.; Zbidi, A.; Amri, M.; Lac, G.; Tabka, Z. Effects of two-month physical-endurance and diet-restriction programmes on lipid profiles and insulin resistance in obese adolescent boys. Diabetes Metab., 2008, 34(6 Pt 1), 595600 .

[12] Balagopal, P.; George, D.; Patton, N.; Yarandi, H.; Roberts, W.L.; Bayne, E.; Gidding, S. Lifestyle-only intervention attenuates the inflammatory state associated with obesity: a randomized controlled study in adolescents. J. Pediatr., 2005, 146(3), 342-348.
[13] Reinehr, T.; Stoffel-Wagner, B.; Roth, C.L. Adipocyte fatty acidbinding protein in obese children before and after weight loss. Metab. Clin. Exp., 2007, 56(12), 1735-1741.

[14] Reinehr, T.; Stoffel-Wagner, B.; Roth, C.L.; Andler, W. Highsensitive C-reactive protein, tumor necrosis factor alpha, and cardiovascular risk factors before and after weight loss in obese children. Metabolism, 2005, 54(9), 1155-1161.

[15] Solbraa, A.K.; Mamen, A.; Resaland, G.K.; Johannessen, J.S.; Ylvisåker, E.; Holme, I.M.; Anderssen, S.M. Level of physical activity, cardiorespiratory fitness and cardiovascular disease risk factors in a rural adult population in Sogn og Fjordane. Nor. Epidemiol., 2011, 20(2), 179-188.

[16] McGavock, J.M.; Torrance, B.D.; McGuire, K.A.; Wozny, P.D.; Lewanczuk, R.Z. Cardiorespiratory fitness and the risk of overweight in youth: the Healthy Hearts Longitudinal Study of Cardiometabolic Health. Obesity (Silver Spring), 2009, 1(9), 18021807.

[17] Ruiz, J.R.; Ortega, F.B.; Martinez-Gomez, D.; Labayen, I.; Moreno, L.A.; De Bourdeaudhuij, I.; Manios, Y.; Gonzalez-Gross, M.; Mauro, B.; Molnar, D.; Widhalm, K.; Marcos, A.; Beghin, L.; Castillo, M.J.; Sjostrom, M. Objectively measured physical activity and sedentary time in European adolescents: the HELENA study. Am. J. Epidemiol., 2011, 174(2), 173-184.

[18] COSI, European Childhood Obesity Surveillance Initiative - Saúde, M.d., Ed. 2009.

[19] Padez, C.; Fernandes, T.; Mourao, I.; Moreira, P.; Rosado, V. Prevalence of overweight and obesity in 7-9-year-old Portuguese children: trends in body mass index from 1970-2002. Am. J. Hum. Biol., 2004, 16(6), 670-678.

[20] Matthews, D.R.; Hosker, J.P.; Rudenski, A.S.; Naylor, B.A.; Treacher, D.F.; Turner, R.C. Homeostasis model assessment: insulin resistance and beta-cell function from fasting plasma glucose and insulin concentrations in man. Diabetologia, 1985, 28(7), 412-419.

[21] Reinehr, T.; Andler, W. Changes in the atherogenic risk factor profile according to degree of weight loss. Arch. Dis. Child., 2004, $89(5), 419-422$.

[22] Ginsberg, H.N. Insulin resistance and cardiovascular disease. $J$. Clin. Investig., 2000, 106(4), 453-458.

[23] Witztum, J.L. Susceptibility of low-density lipoprotein to oxidative modification. Am. J. Med., 1993, 94(4), 347-349.

[24] Byrne, C.D. Triglyceride-rich lipoproteins: are links with atherosclerosis mediated by a procoagulant and proinflammatory phenotype? Atherosclerosis, 1999, 145(1), 1-15.

[25] Packard, C.; Caslake, M.; Shepherd, J. The role of small, dense low density lipoprotein (LDL): a new look. Intern. J. Cardiol., 2000, 74(1), S17-S22.

[26] Lusis, A.J. Atherosclerosis. Nature, 2000, 407(6801), 233-241.

[27] Mertens, A.; Holvoet, P. Oxidized LDL and HDL: antagonists in atherothrombosis. FASEB J., 2001, 15 (12), 2073-2084.

[28] Nofer, J.R.; Kehrel, B.; Fobker, M.; Levkau, B.; Assmann, G.; von Eckardstein, A. HDL and arteriosclerosis: beyond reverse cholesterol transport. Atherosclerosis, 2002, 161(1), 1-16.

[29] Berglund, L.; Ramakrishnan, R. Lipoprotein(a): an elusive cardiovascular risk factor. Arterioscler. Thromb. Vasc. Biol., 2004, 24(12), 2219-2226.

[30] Trommsdorff, M.; Kochl, S.; Lingenhel, A.; Kronenberg, F.; Delport, R.; Vermaak, H.; Lemming, L.; Klausen, I.C.; Faergeman, O.; Utermann, G.; et al., A pentanucleotide repeat polymorphism in the $5^{\prime}$ control region of the apolipoprotein(a) gene is associated with lipoprotein(a) plasma concentrations in Caucasians. J. Clin. Investig., 1995, 96(1), 150-157.

[31] Ouchi, N.; Shibata, R.; Walsh, K. Cardioprotection by adiponectin. Trends Cardiovasc. Med., 2006, 16 (5), 141-146.

[32] Shaibi, G.Q.; Cruz, M.L.; Weigensberg, M.J.; Toledo-Corral, C.M.; Lane, C.J.; Kelly, L.A.; Davis, J. N.; Koebnick, C.; Ventura, E.E.; Roberts, C.K.; Goran, M.I. Adiponectin independently predicts metabolic syndrome in overweight Latino youth. J. Clin. Endocrinol. Metab., 2007, 92(5), 1809-1813. 
[33] Weiss, R.; Dufour, S.; Groszmann, A.; Petersen, K.; Dziura, J.; Taksali, S. E.; Shulman, G.; Caprio, S., Low adiponectin levels in adolescent obesity: a marker of increased intramyocellular lipid accumulation. J. Clin. Endocrinol. Metab., 2003, 88(5), 2014-2018.
[34] Reinehr, T.; Roth, C.; Menke, T.; Andler, W. Adiponectin before and after weight loss in obese children. J. Clin. Endocrinol. Metab., 2004, 89(8), 3790-3794.

[35] Rodgers, A.; Ezzati, M.; Vander Hoorn, S.; Lopez, A.D.; Lin, R.B.; Murray, C.J. Distribution of major health risks: findings from the Global Burden of Disease study. PLoS Med., 2004, l(1), e27.

(C) Nascimento et al.; Licensee Bentham Open.

This is an open access article licensed under the terms of the Creative Commons Attribution Non-Commercial License (http://creativecommons.org/licenses/by-nc/3.0/) which permits unrestricted, non-commercial use, distribution and reproduction in any medium, provided the work is properly cited. 